\title{
Hyaluronidase in hair transplantation
}

Jae Hyun Park, MD Seoul, Korea jay8384@naver.com

\section{Introduction}

The complication that must be avoided in donor harvests with the incisional method in hair transplantation is the situation in which the wound margins do not approximate after the donor strip harvest due to the excessive size of the donor design. If approximation is performed under excessive tension, partial flap necrosis, visible scarring, hypertropic scarring, or donor-hair effluvium may occur. It is a situation that if a hair surgeon has experienced it once, he or she would never want to repeat it.

In order to avoid the problem, it is extremely important to make an appropriate donor design by figuring out the scalp laxity in advance. However, even after doing this, a hair surgeon might still face this unhappy situation.

In most of these cases, the problem is the lack of additional laxity of about 2-3mm. In such situations, I feel hyaluronidase is very useful. Hyaluronidase increases the elasticity of tissue through degradation of hyaluronic acid in dermal collagen.

I conducted the present study to investigate the amount of increased scalp laxity after hyaluronidase injection.

\section{Materials and Methods}

There were 50 study subjects that included 25 men and 25 women. Scalp laxity was measured from the mid-occipital point on the horizontal plane of the upper border of the helix. The Laxometer of Dr. Parsa Mohebi was used for laxity measurement. ${ }^{1}$

After measuring baseline laxity, we mixed hyaluronidase $1 / 2$ ampoule (750 I.U.) with $3 \mathrm{cc}$ saline and injected the mixture in the dermal layer at 10 points with $0.3 \mathrm{cc}$ (75 I.U. per each point). Next, we remeasured the laxity. We again injected hyaluronidase $1 / 2$ ampoule (750 I.U.) that we mixed with $3 \mathrm{cc}$ saline at 10 points in dermal layers with $0.3 \mathrm{cc}$ (75 I.U. per each point). Then we measured the laxity again. In order to prevent changes across positions, we performed measurements with the patient in the same prone position for all measurements.

\section{Results}

Results are shown in the tables below.

Table 1. Laxity measurement $(\mathrm{N}=50)$

\begin{tabular}{|c|c|c|}
\hline $\begin{array}{l}\text { Average laxity } \\
\text { before injection }\end{array}$ & $\begin{array}{l}\text { Laxity after first } \\
\text { injection }\end{array}$ & $\begin{array}{l}\text { Laxity after second } \\
\text { injection }\end{array}$ \\
\hline Upward Downward & Upward Downward & Upward Downward \\
\hline $15.1 \mathrm{~mm} \quad 14.6 \mathrm{~mm}$ & $17.2 \mathrm{~mm} \quad 16.8 \mathrm{~mm}$ & $17.3 \mathrm{~mm} \quad 16.8 \mathrm{~mm}$ \\
\hline
\end{tabular}

Table 2. Increased laxity after hyaluronidase injection

$$
\begin{array}{cl}
\text { Average increased laxity } \\
\text { Upward } & \text { Downward } \\
2.1 \mathrm{~mm} & 2.2 \mathrm{~mm}
\end{array}
$$

The measurement results showed that laxity increased by $2.1 \mathrm{~mm}$ and $2.2 \mathrm{~mm}$ for both upward and downward directions after hyaluronidase 750 I.U. injection. When an additional 750 I.U. was injected, the amount of increase in laxity was almost insignificant.

\section{Discussion}

Hyaluronic acid (HA) is a multifunctional high molecular weight polysaccharide that mainly exists in the extracellular matrix (ECM) of soft connective tissue. Hyaluronidase is an enzyme that degrades hyaluronic acid (HA) and was first introduced by Karl Meyer. ${ }^{2}$

Hyaluronidase has been used in various medical applications. ${ }^{3,4}$ First, by cleaving hyaluronic acid in tissues, it increases membrane permeability, decreases viscosity, and gives injected fluid a more spreading effect. Thus, hyaluronidase is often used in removing edema and bruises after cosmetic surgery. Second, it is used to reduce tissue damage during extravasation of parenteral nutrition solution, antibiotics, and chemotherapeutic agents. Third, hyaluronidase is used in nerve block anesthesia and in retrobulbar anesthesia before ophthalmic surgery to enhance the hypotonic effect of local anesthesia. Fourth, it is used in the treatment of granulomatous hyaluronic acid reactions or unwanted hyaluronic acid misplacement. Finally, it is used in a variety of other ways including orthopedic surgery, internal medicine, anesthesiology, and gynecology.

Hyaluronidase can also be useful in hair transplantation. A useful tip is to use hyaluronidase when the donor margins do not approximate or severe post-operative pain is expected due to excessive wound tension. The application can be done directly during surgery because increase in elasticity shows up within 1 minute of injection. However, a few adverse reactions have been reported including edema associated with hypodermoclysis or dilution with local anesthetics, enhancement of adverse events associated with the co-administered drugs, and urticaria and angioedema in less than $0.1 \%$ of patients. ${ }^{3,5}$ As such, a preliminary skin test using an intradermal injection to observe local wheal and flare reaction within 5 minutes is recommended. ${ }^{3}$

\section{Conclusion}

Hyaluronidase can be useful for closure of donor wounds that are under excessive tension by increasing the elasticity of the scalp.

\section{References}

1. Mohebi, P. The laxometer: increasing the safety of hair tranplants that use a large number of grfts. Hair Transplant Forum Int'l. 2012; 22(6):226-227.

2. Meyer, K. Hyaluronidases. In: P.D. Boyer, Ed., The Enzymes. Academic press: New York; 1971, pp. 307-320.

3. Broody, H.J., Use of hyaluronidase in the treatment of granulomatous hyaluronic acid reactions or unwanted hyaluronic acid misplacement. Dermatol Surg. 2005; 31:8. Part 1.

4. Girish, K.S., and K. Kemparaju. The magic glue hyaluronan and its eraser hyaluronidase: a biological overview. Life Sciences. 2007; 80:1921-1943.

5. Vitrase (hyaluronidase for injection) (package labeling). Irvine (CA): ISTA Phamaceuticals. May 2004. 